\title{
Determination of Isotianil in Brown Rice and Soil Using Supercritical Fluid Extraction and Gas Chromatography/Mass Spectrometry
}

\author{
Tatsuo Yoshida, ${ }^{*}, * *+$ Asami Itou, ${ }^{*}$ Rise Yamamoto, ${ }^{*}$ Toshiaki Tobino, ${ }^{*}$ Hiroshi Murakawa, ${ }^{*}$ and \\ Kei ToDA**
}

*Kumamoto Prefectural Institute of Public Health and Environmental Science, 1240-1 Kurisaki, Uto 869-0425, Japan

**Department of Chemistry, Kumamoto University, 2-39-1 Kurokami, Chuo, Kumamoto 860-8555, Japan

\begin{abstract}
Isotianil (3,4-dichloro-2'-cyano-1,2-thiazole-5-carboxanilide) is a new plant-activating pesticide. Usage of the pesticide was approved for rice fields in 2010 and its production increased 400 times $\left(2 \times 10^{4} \mathrm{~kg}\right)$ in the next year. In this work, a method for determining isotianil in brown rice and rice field soil was investigated for the first time. Isotianil was extracted by supercritical fluid extraction and measured by gas chromatography/mass spectrometry. Isotianil was successfully analyzed with good recoveries $(95.1-99.3 \%)$ even from soil samples with strong adsorption of pesticides.
\end{abstract}

Keywords Isotianil, pesticide, supercritical fluid extraction, gas chromatography/mass spectrometry, brown rice, soil

(Received July 1, 2013; Accepted August 5, 2013; Published September 10, 2013)

\section{Introduction}

Isotianil (3,4-dichloro-2'-cyano-1,2-thiazole-5-carboxanilide) is a novel pesticide that acquired Japanese pesticide registration approval for rice fields in 2010. This pesticide is categorized as a "plant activator", i.e. it does not show direct antimicrobial activity against pathogenic microbes but instead prevents the outbreak of disease by inducing systemic acquired resistance in the host plant. Isotianil demonstrates preventive effects against serious rice diseases such as rice blast (Magnaporthe grisea), bacterial leaf blight (Xanthomonas oryzae pv. oryzae), and a wide range of other rice diseases. The outstanding characteristics of isotianil are that its effect lasts for a long period even at low dosage without having significant effect on non-target organisms. Isotianil has therefore recently attracted much attention in the field of agriculture. Isotianil is registered as a usable pesticide in South Korea as well as in Japan, and the use of isotianil will be extended to other rice-growing countries. Usage of isotianil was approved in 2010 in Japan and production increased 400 times to $2 \times 10^{4} \mathrm{~kg}$ in the following year. Therefore, an analytical method for isotianil is urgently required as an official analytical method has not yet been announced by the Japanese Ministry of Health, Labour and Welfare and no analytical method for isotianil has been reported in existing literature.

Recently, supercritical fluid extraction (SFE) has become popular for the rapid analysis of many types of pesticides. SFE uses a fluid in the supercritical state; the fluid has high permeability, like gaseous species, and good solvents for pesticides, like organic solvents, have been used to extract pesticides from agricultural products ${ }^{1,2}$ and dioxins from soils. ${ }^{3}$ We previously investigated SFE of pesticides from agricultural

† To whom correspondence should be addressed. E-mail: yoshida-t-dp@ pref.kumamoto.lg.jp products, soils, ${ }^{4}$ and wastewater, ${ }^{5}$ and of polychlorinated biphenyls from solid wastes. ${ }^{6}$ Pesticides, in various kinds of agricultural products that are strongly adsorbed to sediment matrixes, were successfully extracted using the described SFE method. Extraction using SFE is simpler and less time consuming compared to other extraction methods such as Soxhlet extraction.

Analysis of pesticide residues in agricultural products contributes to the safety of food products and prevents health problems caused by ingestion of excess pesticides. Moreover, investigation of residual pesticides is useful in understanding the environmental dynamics of pesticides. In this work, an analytical method for isotianil was investigated for the first time, and a simple method was established using SFE followed by gas chromatography/mass spectrometry (GC/MS).

\section{Experimental}

\section{Standard solution}

A stock solution of isotianil $\left(1000 \mathrm{mg} \mathrm{L}^{-1}\right)$ was prepared by dissolving isotianil (Sigma-Aldrich, St. Louis, MO) in acetone, and standard test solutions were obtained by diluting the stock solution with acetone. For calibrations, matrix-matched standard solutions were prepared by diluting the stock solution with extracts from isotianil-free brown rice and soil.

\section{Sample treatment}

Recovery tests were performed using brown rice and soil samples obtained from an agricultural field where isotianil had not been used before. The brown rice was pulverized using a food cutter into particles that could be passed through a $425-\mu \mathrm{m}$ standard sieve. The texture of the soil was a loam containing $7.4 \%(\mathrm{w} / \mathrm{w})$ organic carbon, and its $\mathrm{pH}$ was 6.2 . The soil was homogenized by hand mixing, dried at room temperature for 


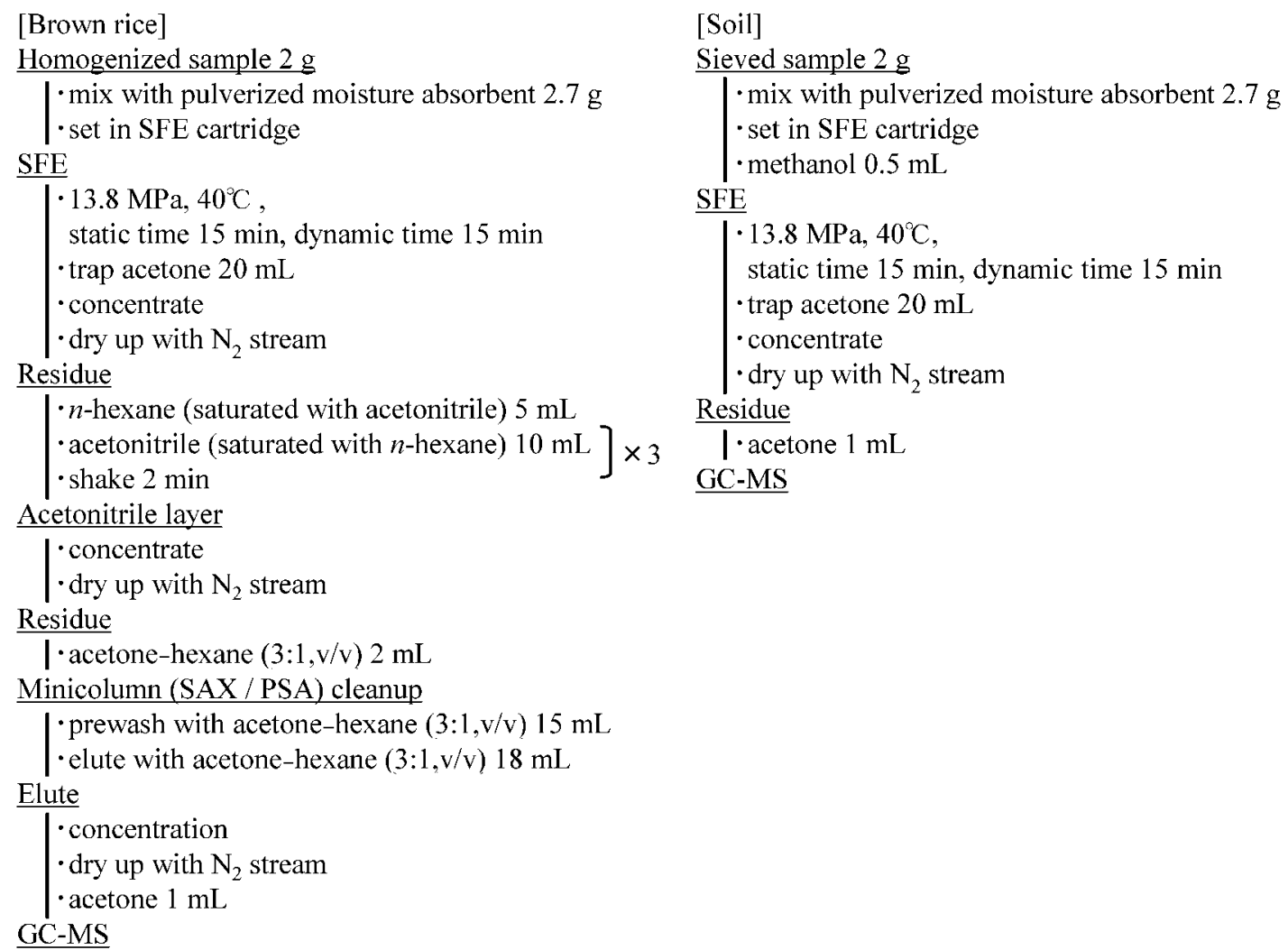

Fig. 1 Treatment of brown rice and soil samples for GC/MS analysis.

$5 \mathrm{~d}$, and sieved to a particle size of $<2 \mathrm{~mm}$. Isotianil was added to the samples to give concentrations of $0.01,0.05$ and $0.3 \mathrm{mg} \mathrm{kg}^{-1}$ for recovery examinations.

\section{SFE}

The instrument used for SFE was an SFX1220 (Teledyne ISCO, Lincoln, NE) equipped with a 10-mL hand-tight SFE vessel. Carbon dioxide was introduced as the SFE fluid into the vessel, which was maintained at $13.8 \mathrm{MPa}$ and $40^{\circ} \mathrm{C}$, to perform static extraction for $15 \mathrm{~min}$ and subsequent dynamic extraction with a fluid flow of $2 \mathrm{~mL} \mathrm{~min}^{-1}$ for $15 \mathrm{~min}$. The restrictor temperature was maintained at $60^{\circ} \mathrm{C}$.

The extraction process is schematically shown in Fig. 1. The sample ( $2 \mathrm{~g}$ ) was mixed with $2.7 \mathrm{~g}$ of moisture absorbent (Chem Tube-hydromatrix, Agilent, Santa Clara, CA), which was finely powdered beforehand with a food cutter, and placed in the extraction vessel. For soil analysis, $0.5 \mathrm{~mL}$ of methanol was added to the mixed sample as modifier. The extracted species were trapped with $20 \mathrm{~mL}$ of acetone, and the acetone solution was then concentrated using a rotary evaporator and dried with a nitrogen stream. The concentrated extract from the soil sample was diluted with acetone to a volume of $1 \mathrm{~mL}$ for GC/MS analysis.

\section{Purification of brown rice extracts}

While extracts from soil were analyzed without further treatment, purification was necessary for brown rice analysis because brown rice contains lipids that interfere with GC analysis. The brown rice extract was mixed with $5 \mathrm{~mL}$ of $n$-hexane saturated with acetonitrile and purified as follows. The sample solution was mixed with $10 \mathrm{~mL}$ of acetonitrile saturated with $n$-hexane and mechanically shaken for 2 min and then the acetonitrile layer was transferred to a flask. This procedure was repeated three times, and the obtained acetonitrile layer, $\sim 30 \mathrm{~mL}$ in total, was concentrated using a rotary evaporator and then dried with a nitrogen stream. The residue was dissolved in 2-mL mixture of acetone and $n$-hexane $(3: 1)$ and placed on top of a $20-\mathrm{mL}$ mini-column packed with $500 \mathrm{mg}$ of strong anion-exchange resin (SAX) and $500 \mathrm{mg}$ of $N$-propylethylenediamine (PSA) (InertSep SAX/PSA, GL Sciences, Tokyo, Japan), which was treated in advance with $15 \mathrm{~mL}$ of acetone $/ n$-hexane mixture (3:1). The analytes were eluted with $18 \mathrm{~mL}$ of the same solvent mixture. Acetone and $n$-hexane in the eluent were removed by evaporation and the residue was dissolved in $1 \mathrm{~mL}$ of acetone for GC/MS analysis. For calibration samples, matrix-matched standard solutions were prepared using solutions of extracts from contaminant-free samples.

\section{GC/MS measurements}

The GC/MS instrument used was an Agilent7890/5975C (Agilent) equipped with a capillary column $(0.25 \mathrm{~mm}$ i.d. $\times$ $30 \mathrm{~m}, 0.25 \mu \mathrm{m}$ thickness, HP-5MS, Agilent). The extract sample $(4 \mu \mathrm{L})$ was injected using the pulsed splitless mode into an injector heated to $250^{\circ} \mathrm{C}$. The column oven temperature was kept at $80^{\circ} \mathrm{C}$ for $2 \mathrm{~min}$ and then increased to $180^{\circ} \mathrm{C}$ and up to $300^{\circ} \mathrm{C}$ at rates of 30 and $10^{\circ} \mathrm{C} \mathrm{min}{ }^{-1}$, respectively; a temperature of $300^{\circ} \mathrm{C}$ was maintained for $12 \mathrm{~min}$. The analyte was ionized by electron ionization using an ion source heated to $230^{\circ} \mathrm{C}$, and quantitative and qualitative measurements were performed by selected ion monitoring (SIM) at $\mathrm{m} / z, 297$ and 180, respectively, using a quadrupole ion separator heated to $150^{\circ} \mathrm{C}$. 

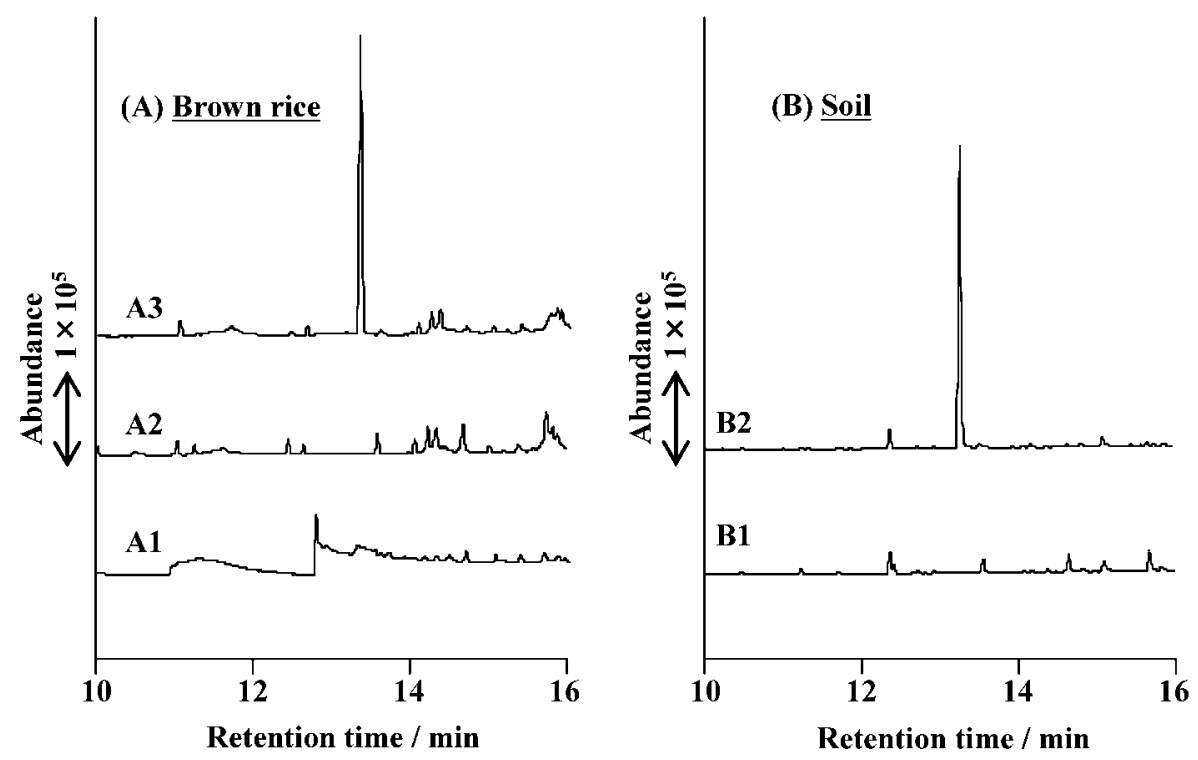

Fig. 2 Chromatograms (SIM, $m / z$ 297) obtained for extracts from brown rice (panel A, A1 - 3) and soil (panel B, B1 - 2). Chromatograms for brown rice were obtained without purification (A1), after purification (A2) and with isotianil spiking after purification (A3). For soil, chromatograms B1 and B2 were obtained without and with isotianil spiking, respectively.

\section{Results and Discussion}

\section{Chromatographic performance}

The calibration curves obtained for matrix-matched standard solutions were straight $\left(R^{2}>0.999\right)$ in the range $2-600 \mu \mathrm{g} \mathrm{L}-1$, and this linear range corresponded to $1-300 \mu \mathrm{g} \mathrm{kg}-1$ for $2 \mathrm{~g}$ of solid sample. Typical chromatograms obtained are shown in Fig. 2. Isotianil appeared in the chromatograms at $13.3 \mathrm{~min}$ of retention time. In the chromatogram for brown rice (A1 in Fig. 2), the baseline was relatively high and interference peaks were observed, probably due to high lipid content in brown rice. However, good baseline and isotianil peak were obtained when the purification process was adopted. On the other hand, purification was not necessary in the case of soil analysis as shown in panel B of Fig. 2. The limits of detection (LOD, $S / N=3$ ) and limits of determination (LOQ, $S / N=10$ ) were 0.23 and $0.77 \mu \mathrm{g} \mathrm{kg}^{-1}$, respectively, for brown rice, and 0.19 and $0.63 \mu \mathrm{g} \mathrm{kg}^{-1}$, respectively, for soil samples. The method is therefore sufficiently sensitive to detect $1 / 100$ of the maximum residue limit of isotianil in brown rice $\left(300 \mu \mathrm{g} \mathrm{kg}^{-1}\right)$ specified by the Japanese Ministry of Health, Labour and Welfare.

\section{Extraction pressure}

The effect of SFE pressure was investigated for extraction of isotianil. As carbon dioxide becomes supercritical above $7.38 \mathrm{MPa}$, the pressure was set at 10.3, 13.8, 17.2, and 20.7 $\mathrm{MPa}$, corresponding to $1500,2000,2500$ and $3000 \mathrm{psi}$, respectively. Table 1 shows the recoveries achieved for brown rice and soil at different pressures. The same trend was observed for brown rice and soil; recovery was low at $10.3 \mathrm{MPa}$ and increased at 13.8 MPa. The recovery decreases with further increases in pressure above $13.8 \mathrm{MPa}$. Higher pressure accelerates dissolution of analyte compounds, but too much pressure interferes with diffusion of analyte molecules. ${ }^{7}$ It was decided that the optimal pressure for isotianil extraction was 13.8 MPa. Good recovery, 98.0\%, was obtained for extraction from brown rice. However, the recovery was only $76.7 \%$ from
Table 1 Effect of pressure and modifier on SFE from isotianilspiked samples

\begin{tabular}{cccc}
\hline \multirow{2}{*}{$\begin{array}{c}\text { Extraction } \\
\text { pressure/MPa }\end{array}$} & $\begin{array}{c}\text { Modifier } \\
\left(\log P_{\text {ow }} \mathrm{a}\right) / \mathrm{mL}\end{array}$ & \multicolumn{2}{c}{ Recovery,$\%(n=3)$} \\
\cline { 3 - 4 } & - & Brown rice & Soil \\
\hline 10.3 & - & $49.7 \pm 4.5$ & $33.3 \pm 1.6$ \\
13.8 & - & $98.0 \pm 1.7$ & $76.7 \pm 3.5$ \\
17.2 & - & $79.0 \pm 3.6$ & $64.2 \pm 3.9$ \\
20.7 & Ethyl acetate $(0.73) / 0.5$ & - & $66.7 \pm 5.1$ \\
13.8 & Acetone $(-0.24) / 0.5$ & - & $89.1 \pm 6.0$ \\
13.8 & Methanol $(-0.82) / 0.25$ & - & $85.5 \pm 2.1$ \\
13.8 & Methanol $(-0.82) / 0.5$ & $95.4 \pm 3.1$ & $98.1 \pm 3.9$ \\
13.8 & Methanol $(-0.82) / 1.0$ & - & $96.0 \pm 3.5$ \\
13.8 & Methanol $(-0.82) / 1.5$ & - & $96.4 \pm 2.3$ \\
13.8 & M.th &
\end{tabular}

a. Octanol-water partition coefficient.

b. Recovery was estimated at $0.05 \mathrm{mg} \mathrm{kg}^{-1}$ concentration level.

soil, even at the optimal pressure. Isotianil was probably strongly adsorbed onto organic matter contained in the soil.

\section{Modifier in soil extraction}

To overcome the problems associated with SFE from soil samples, addition of a modifier was investigated. Polar organic solvents make carbon dioxide polar, improving the extraction efficiency. ${ }^{8-10}$ Ethyl acetate, acetone, and methanol were tested as modifiers. The results for different modifiers are listed in Table 1 with their octanol-water partition coefficients, $P_{\text {ow. }}$. The extraction efficiency without a modifier was $76.7 \pm 3.5 \%$, and increased with the addition of a modifier. Interestingly, the effect of the modifier improved with increasing modifier polarity. When $0.5 \mathrm{~mL}$ of each modifier was added to soil samples, the recoveries achieved were $66.7 \%$ with ethyl acetate $\left(\log P_{\mathrm{ow}}=0.73\right), 89.1 \%$ with acetone $\left(\log P_{\mathrm{ow}}=-0.24\right)$, and $98.1 \%$ with methanol $\left(\log P_{\mathrm{ow}}=-0.82\right)$. Accordingly, methanol was the best modifier. Next, recovery was examined with 
Table 2 Performance of the analytical method for isotianil in brown rice and soil

\begin{tabular}{lcccc}
\hline Sample & $\begin{array}{c}\text { Concentration/ } \\
\mathrm{mg} \mathrm{kg}^{-1}\end{array}$ & $\begin{array}{c}\text { Recovery, } \\
\%(n=6)\end{array}$ & $\begin{array}{c}\mathrm{LOD} / \\
\mu \mathrm{kg}^{-1} \\
(S / N=3)\end{array}$ & $\begin{array}{c}\mathrm{LOQ} / \\
\mu \mathrm{g} \mathrm{kg}^{-1} \\
(S / N=10)\end{array}$ \\
\hline Brown rice & 0.01 & $97.0 \pm 2.8$ & 0.23 & 0.77 \\
& 0.05 & $99.3 \pm 2.8$ & & \\
Soil & 0.3 & $96.1 \pm 1.9$ & & \\
& 0.01 & $95.2 \pm 1.8$ & 0.19 & 0.63 \\
& 0.05 & $95.1 \pm 2.6$ & & \\
& 0.3 & $95.4 \pm 2.5$ & & \\
\hline
\end{tabular}

different amounts of methanol. While the lowest recovery $(85.5 \%)$ was obtained with $0.25 \mathrm{~mL}$ of methanol, recovery was good $(96.0-98.1 \%)$ between 0.5 and $1.5 \mathrm{~mL}$ of methanol as shown in Table 1. We decided to use $0.5 \mathrm{~mL}$ of methanol as a modifier for the extraction of isotianil from soil samples. In the case of brown rice, quantitative extraction was obtained in both cases with and without the modifier, and a modifier was not used for further experiments for brown rice samples.

\section{Analysis of brown rice and soil}

It is known that analytical results may be affected by ion suppression or ion enhancement during the ionization process in mass analysis if the sample has a high content of matrix compounds. ${ }^{11-13}$ Ion enhancement of $10 \%$ was observed for isotianil extracted from brown rice and soil in GC/MS analysis. The quantification of isotianil should therefore be performed using matrix-matched standard solutions prepared from isotianil-free brown rice and soil.

The performance of the analytical method was examined using the optimal conditions, and the results are shown in Table 2. Isotianil was added at concentrations of $0.01,0.05$ and $0.3 \mathrm{mg} \mathrm{kg}^{-1}$ to examine the SFE-GC/MS performance, and each sample was analyzed six times to determine intraday deviations. The recoveries were $96.1-99.3 \%$ for the brown rice samples and $95.1-95.4 \%$ for the soil samples, respectively. No obvious difference was observed among the three concentration tests, and good recoveries were obtained for all examinations. In addition to the intraday tests, inter-day examinations were performed; the recovery deviations were $5.4 \%$ for the brown rice and $5.6 \%$ for the soil sample examined for $6 \mathrm{~d}$ with $0.05 \mathrm{mg} \mathrm{kg}^{-1}$ of isotianil added to the samples. Good inter-day deviations were obtained for both brown rice and soil.

\section{Conclusions}

A simple and rapid method for the determination of a new plant-activating pesticide, isotianil, in brown rice and soil was established, which comprised SFE and GC/MS analysis. Sufficient extraction was achieved from brown rice and soil samples without and with a modifier. This method is expected to be applicable to other pesticide residues in brown rice and soil samples for the simultaneous analysis of multiple pesticides. The proposed method is a simple and rapid analytical tool for pesticide determination and is expected to contribute greatly to ensuring the safety of agricultural products with the development of new pesticides.

\section{References}

1. M. Saka, K. Iijima, Y. Odanaka, and Y. Kato, J. Pestic. Sci., 1998, 23, 414.

2. S. Nemoto, J. Pestic. Sci., 2007, 32, 328.

3. T. Miyawaki, A. Kawashima, and K. Honda, Bunseki Kagaku, 2003, 52, 989.

4. T. Yoshida, H. Murakawa, K. Fukushima, H. Yoshimoto, and T. Tobino, Bunseki Kagaku, 2009, 58, 931.

5. H. Chikushi, K. Hirota, N. Yoshida, T. Edamura, and K. Toda, Talanta, 2009, 80, 738.

6. H. Chikushi, Y. Fujii, and K. Toda, J. Chromatogr., A, 2012, 1256, 267.

7. V. Librando, O. Hutzinger, G. Tringali, and M. Aresta, Chemosphere, 2004, 54, 1189.

8. A. Koinecke, R. Kreuzig, and M. Bahadir, J. Chromatogr., A, 1997, 786, 155.

9. M. L. Jeong and D. J. Chesney, J. Supercrit. Fluids, 1999, $16,33$.

10. J. R. Forero-Mendieta, H. I. Castro-Vargas, F. ParadaAlfonso, and J. A. Guerrero-Dallors, J. Supercrit. Fluids, 2012, 68, 64.

11. H. Kobayashi, Bunseki Kagaku, 2009, 58, 985.

12. T. Yoshida, H. Hamada, H. Murakawa, H. Yoshimoto, T. Tobino, and K. Toda, Anal. Sci., 2012, 28, 179.

13. T. Yoshida, H. Murakawa, and K. Toda, J. Pestic. Sci., 2013, 38, 27. 